OPEN ACCESS

Edited by:

Xin Qi,

Case Western Reserve University School of Medicine, United States

Reviewed by:

Bing Zhou,

Tsinghua University, China Wladyslaw-Lason, Institute of Pharmacology PAS in Krakow, Poland

*Correspondence: Shao-Peng Lin linsp@qq.com Shao-Hua Yang shaohua.yang@unthsc.edu

Received: 23 January 2018 Accepted: 03 April 2018 Published: 20 April 2018

Citation: Lin S-P, Li W, Winters A, Liu R and Yang S-H (2018) Artemisinin Prevents

Glutamate-Induced Neuronal Cell Death Via Akt Pathway Activation.

Front. Cell. Neurosci. 12:108. doi: 10.3389/fncel.2018.00108

\section{Artemisinin Prevents Glutamate-Induced Neuronal Cell Death Via Akt Pathway Activation}

\author{
Shao-Peng Lin ${ }^{1,2 *}$, Wenjun Li ${ }^{1}$, Ali Winters ${ }^{1}$, Ran Liu ${ }^{1}$ and Shao-Hua Yang ${ }^{1 *}$ \\ ${ }^{1}$ Department of Pharmacology and Neuroscience, University of North Texas Health Science Center, Fort Worth, TX, \\ United States, ${ }^{2}$ Department of Emergency, The Second Affiliated Hospital of Guangzhou Medical University, \\ Guangzhou, China
}

Artemisinin is an anti-malarial drug that has been in use for almost half century. Recently, novel biological effects of artemisinin on cancer, inflammation-related disorders and cardiovascular disease were reported. However, neuroprotective actions of artemisinin against glutamate-induced oxidative stress have not been investigated. In the current study, we determined the effect of artemisinin against oxidative insult in HT-22 mouse hippocampal cell line. We found that pretreatment of artemisinin declined reactive oxygen species (ROS) production, attenuated the collapse of mitochondrial membrane potential induced by glutamate and rescued HT-22 cells from glutamate-induced cell death. Furthermore, our study demonstrated that artemisinin activated Akt/Bcl2 signaling and that neuroprotective effect of artemisinin was blocked by Akt-specific inhibitor, MK2206. Taken together, our study indicated that artemisinin prevented neuronal HT-22 cell from glutamate-induced oxidative injury by activation of Akt signaling pathway.

Keywords: artemisinin, Akt, oxidative stress, apoptosis, neuroprotection

\section{INTRODUCTION}

Artemisinin, first discovered in 1970s, has pioneered a new era for the treatment of malaria and saved millions malarial patients worldwide (Guo, 2016). Although the precise mechanism underlying its anti-malaria effect is still not clear, artemisinin and its derivatives are considered prodrugs that generate carbon-centered free radicals or reactive oxygen species (ROS), which further alkylate key parasite proteins and results in the death of parasite (Cui and Su, 2009; Ismail et al., 2016). Furthermore, artemisinin has been indicated to interact with FADH and/or other parasite flavoenzymes, hence impair parasite redox homeostasis and generation of ROS (Haynes et al., 2010). Paradoxically, antioxidant activity of artemisinin has also been demonstrated (Kim et al., 2015). Artemisinin has been found to protect retinal neuronal cells against oxidative stress (Yan et al., 2017).

Oxidative stress refers to the imbalance between ROS production and antioxidant defense which has been found to be involved in aging and aging-related neurodegenerative disorders (Lin et al., 2016; Sozen and Ozer, 2017; Vida et al., 2017). Correspondently, antioxidant has been an attractive approach for the treatment of neurodegenerative diseases (Xie et al., 2013; Lin et al., 2016). Artemisinin could cross the blood-brain barrier (BBB) without obvious toxicity in the central nervous system, implying favorable advantages in the treatment of neurological disorders (Zuo et al., 2016). However, the effect of artemisinin on oxidative stress in brain cells has not been 
fully investigated. In the present study, we determined the neuroprotective effect of artemisinin on glutamate-induced oxidative injury in HT-22 hippocampal cell line. Our results indicated that artemisinin could prevent neuronal HT-22 cell from glutamate-induced oxidative damage potentially via the activation of Akt pathway.

\section{EXPERIMENTAL PROCEDURES}

\section{Cell Culture}

HT-22 cells, a hippocampal cell line, were maintained in Dulbecco's Modified Eagle's Medium (DMEM; HyClone, USA) supplemented with $10 \%$ fetal calf serum (FBS; HyClone, USA), $50 \mathrm{IU} / \mathrm{ml}$ penicillin and $50 \mu \mathrm{g} / \mathrm{ml}$ streptomycin (Sigma-Aldrich, USA) in a humidified incubator with $5 \% \mathrm{CO}_{2}$ at $37^{\circ} \mathrm{C}$. Cells at passage $10-20$ were adjusted to $3 \times 10^{4} / \mathrm{ml}$ and were plated in 12-well or 96-well cell culture plates (Cellstar, Greiner Bio-One $\mathrm{GmbH})$. At $24 \mathrm{~h}$ after seeding, adherent cells were used for all of the experiments. L-Glutamic acid (CAS No. 138-15-8, SigmaAldrich, USA) and artemisinin (CAS No. 63968-64-9, SigmaAldrich, USA) were purchased from Sigma Aldrich. MK2206
(CAS No. 1032350-13-2) was purchased from Selleck (Houston, TX, USA).

\section{Cell Viability Assay}

Cell viability was assessed using Calcein-AM assay (Anaspec, Fremont, CA, USA) according a protocol modified from our previous publication (Ryou et al., 2015). In brief, cells were washed with phosphate-buffered saline (PBS, $\mathrm{pH} 7.0$ ) and incubated with $1 \mu \mathrm{g} / \mathrm{ml}$ Calcein-AM for $10 \mathrm{~min}$ at $37^{\circ} \mathrm{C}$. Fluorescence was determined using a Tecan Infinite F200 plate reader (Maennedorf, Switzerland) with 485/530$\mathrm{nm}$ excitation/emission. The percentage of cell viability was normalized to the control group.

For Calcein-AM/PI double staining, HT-22 cells were incubated in PBS containing $1 \mu \mathrm{g} / \mathrm{ml}$ Calcein-AM and $5 \mu \mathrm{g} / \mathrm{ml}$ propidium iodide (PI, BD Biosciences) at $37^{\circ} \mathrm{C}$ for $15 \mathrm{~min}$. After washing with PBS, cells were observed by a fluorescence microscope (Axio Observer Z1; Carl Zeiss AG, Germany).

For flow cytometry analysis, floating cells and adherent cells were collected and stained with PI and Annexin V (BD Biosciences) according to the manufacture's instruction. And the cells were analyzed by a BD flow cytometry (BD Biosciences).
A

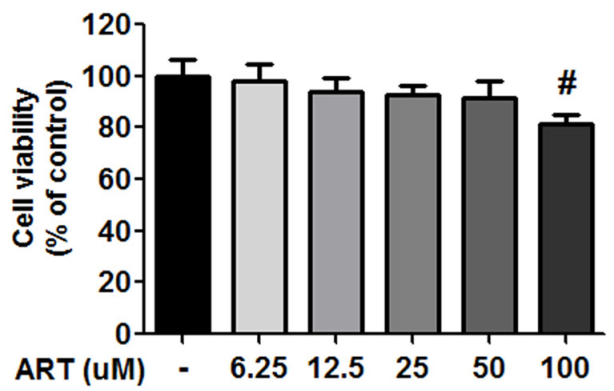

C

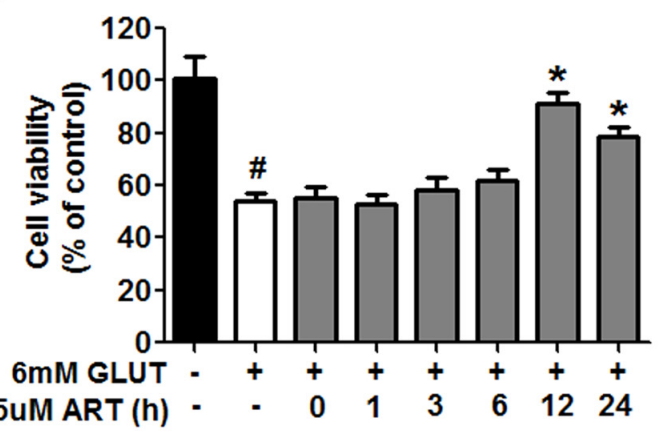

B

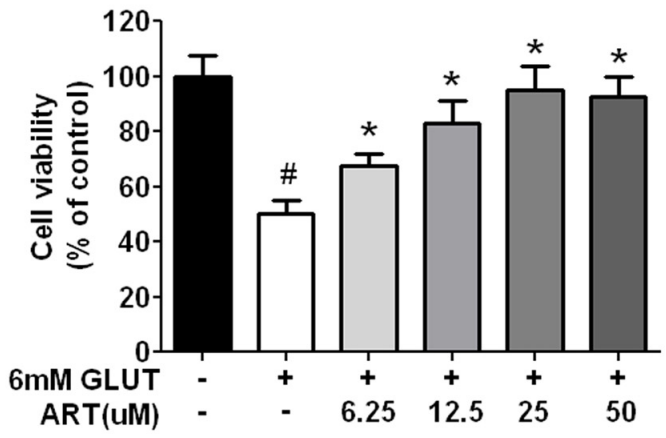

CON

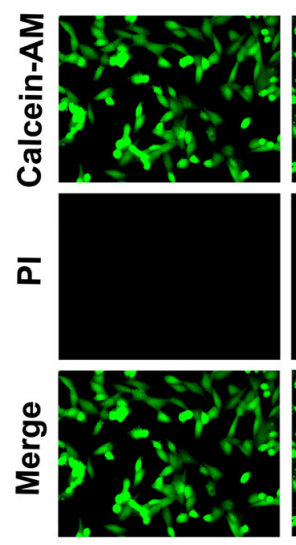

GLUT
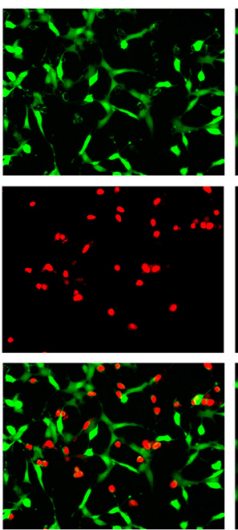

GLUT+ART

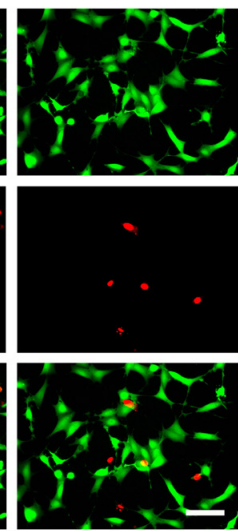

FIGURE 1 | Pretreatment with artemisinin protected HT-22 cells against glutamate-induced cell death. (A) Artemisinin concentration between $6.25-50$ $\mu$ M did not cause any cytotoxicity in HT-22 cells. (B) Pretreatment with artemisinin for $12 \mathrm{~h}$ significantly attenuated glutamate-induced HT-22 cell death in a dose-dependent manner. (C) No protective effect against glutamate-induced toxicity was observed when $25 \mu \mathrm{M}$ artemisinin was pretreated less than $12 \mathrm{~h}$. (D) Calcein-AM/PI double staining showed that pretreatment with $25 \mu \mathrm{M}$ artemisinin for $12 \mathrm{~h}$ attenuated glutamate-induced HT-22 cell death. Scale bar $=100 \mu \mathrm{m}$. ${ }^{\#} p<0.05$ vs. CON group, ${ }^{*} p<0.05$ vs. GLUT group. 


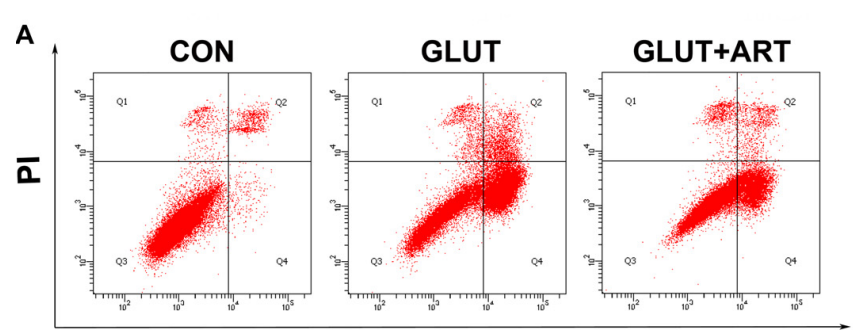

Annexin V

C
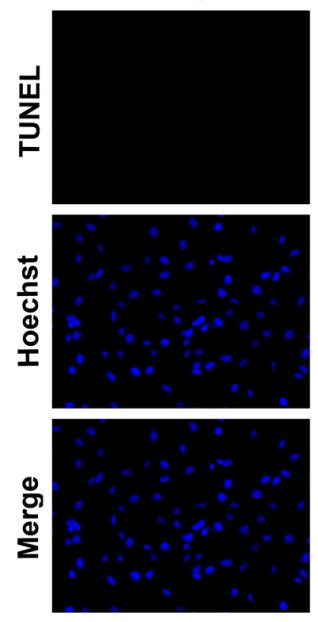
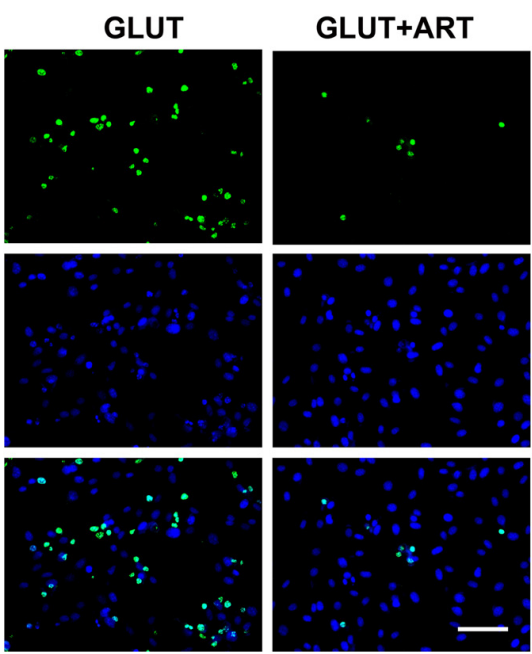

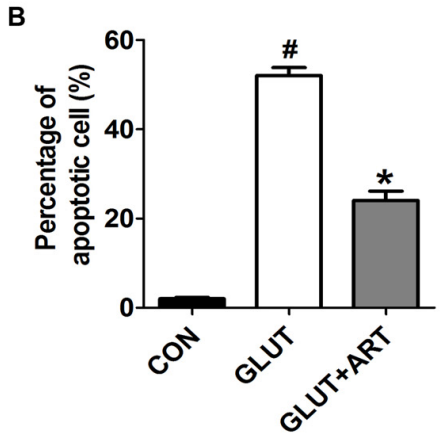

D

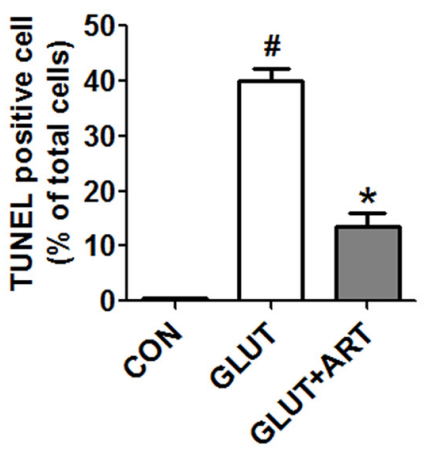

FIGURE 2 | Artemisinin inhibited the glutamate-induced apoptosis in HT-22 cells. (A,B) Flow cytometry analysis indicated the anti-apoptotic effect of artemisinin. (C,D) TUNEL assay manifested that artemisinin attenuated glutamate-induced cell apoptosis significantly. Scale bar $=100 \mu \mathrm{m}$. ${ }^{\#} p<0.05$ vs. CON group, $* p<0.05$ vs. GLUT group.

\section{Reactive Oxygen Species Measurements}

Intracellular and mitochondrial ROS production were assessed by a fluorometric assay using $2^{\prime}, 7^{\prime}$-dichlorofluorescein diacetate (H2DCFDA; Invitrogen, USA) and MitoTracker Red CMXRos (Invitrogen, USA), respectively. After $12 \mathrm{~h}$ treatment of artemisinin and followed by $12 \mathrm{~h}$ treatment of glutamate, cells were incubated in $10 \mu \mathrm{mol} / \mathrm{L}$ H2DCFDA or $0.25 \mu \mathrm{mol} / \mathrm{L}$ MitoTracker Red CMXRos for $30 \mathrm{~min}$ at $37^{\circ} \mathrm{C}$. The fluorescence was then observed via a fluorescence microscope. The fluorescence was detected with 530/485-nm and 579/599-nm excitation/emission wave lengths.

\section{Mitochondrial Membrane Potential ( $\Delta \Psi \mathrm{m})$ Measurement}

The mitochondrial membrane potential was detected using Tetramethylrhodamine, Ethyl Ester (TMRE) mitochondrial membrane potential assay kit (Abcam, USA). Cells were loaded with $20 \mathrm{nM}$ of TMRE working solution for $20 \mathrm{~min}$ at $37^{\circ} \mathrm{C}$. The fluorescent images were observed and obtained on a Zeiss fluorescence microscope. Fluorescence intensity was measured using a Tecan Infinite F200 plate reader (Maennedorf, Switzerland) with 594/575-nm excitation/emission.

\section{Immunocytochemistry and TUNEL Staining}

Cells were fixed in BD Cytofix/Cytoperm solution (BD Biosciences) and permeabilized using $0.1 \%$ Triton-X. The cells were incubated overnight in primary antibody for pAkt (Cell signaling technology, 1:50) followed by staining with Alexa Fluor 488-conjugated goat anti-rabbit IgG (Thermo Scientific, 1:500). Then the cells were further incubated with $0.5 \mathrm{mg} / \mathrm{mL}$ DAPI for nuclei. Images were obtained using a LSM 410 confocal microscope (Zeiss, Thornwood, NY, USA).

The fragmentation of genomic DNA was detected by in situ staining of DNA ends with TdT-mediated dUTP nick end labeling (TUNEL; Progega, USA) following the manufacturer's instruction. Briefly, incubation buffer containing Equlibration Buffer, Nucleotide Mix and rTdT Enzyme was incubated for $2 \mathrm{~h}$ at $37^{\circ} \mathrm{C}$ in the dark. Hoechst 33342 staining was used to count the total number of nuclei. Images were taken with a fluorescence microscope.

\section{Western Blot Analysis}

Western blot analysis was carried out using a protocol modified from our previous publication (Xie et al., 2013). In briefly, cell 

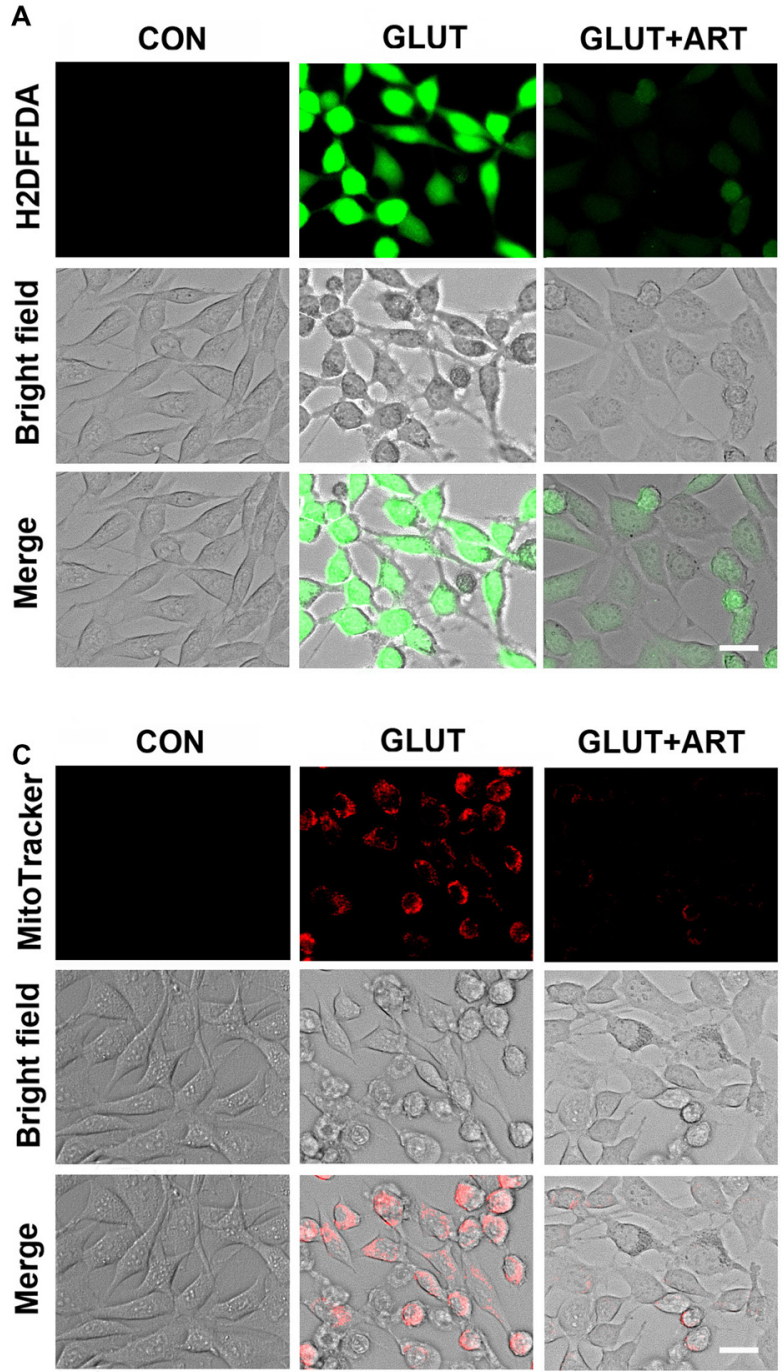

B

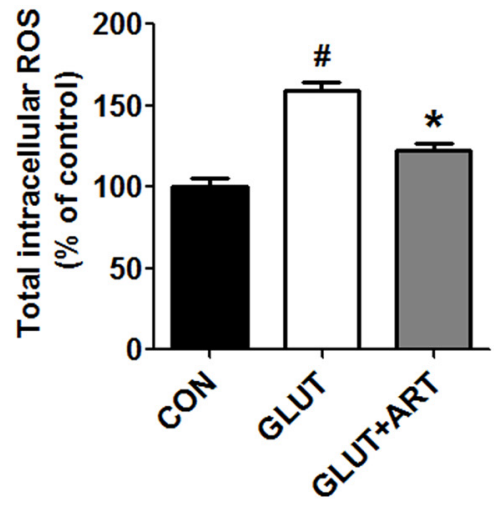

D

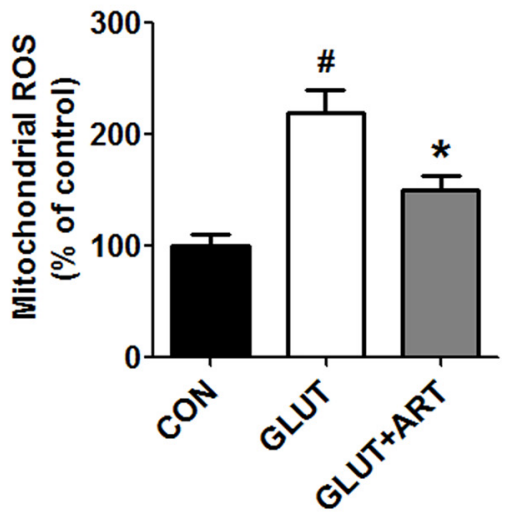

FIGURE 3 | Artemisinin decreased glutamate-induced oxidative stress in HT-22 cells. (A,B) Artemisinin reduced the increase of glutamate-induced total intracellular reactive oxygen species (ROS). (C,D) Artemisinin decreased the elevation of glutamate-induced mitochondrial ROS. Scale bar $=20 \mu \mathrm{m}$. ${ }^{\#} p<0.05$ vs. CON group, ${ }^{*} p<0.05$ vs. GLUT group.

lysate were prepared by homogenization in RIPA buffer $(20 \mathrm{mM}$ Tris- $\mathrm{HCl}, \mathrm{pH}$ 7.5, $150 \mathrm{mM} \mathrm{NaCl}, 1$ mM Na2EDTA, 1 mM EGTA, $1 \% \mathrm{NP}-40$ and $1 \%$ sodium deoxycholate) including phosphatase and protease inhibitor for $20 \mathrm{~min}$ on ice. Proteins were loaded onto $8 \%-12 \%$ SDS-PAGE gel and electrophoresis was performed. Protein samples were transferred to nitrocellulose membranes and incubated with primary antibody overnight at $4^{\circ} \mathrm{C}$ for Phospho-Akt (Ser473; D9E) $\mathrm{XP}^{\circledR}$ Rabbit mAb (Cell signaling technology, 1:2000), Akt (pan; C67E7) Rabbit $\mathrm{mAb}$ (Cell signaling technology, 1:1000), Bcl-2 (Cell signaling technology, 1:1000), Bax (Cell signaling technology, 1:1000), Caspase-3 (Cell signaling technology, 1:1000), PARP (Cell signaling technology, 1:1000) and $\beta$-Actin (C4; Santa Cruz Biotech, 1:2000) antibody. After being washed three times with PBST, membranes were incubated with horseradish peroxidase-conjugated secondary antibodies for $1 \mathrm{~h}$ at room temperature. Membranes were developed with Super Signal West Pico Chemiluminescent Substrate (Thermo Scientific, USA). The optical density of the target protein bands were measured using a Biospectrum 500 imaging system (Ultraviolet Products, Upland, CA, USA).

\section{Statistical Analysis}

Graph Pad Prism 5 was used for statistical analysis. The experiments were carried out at least in triplicate, and all data were presented as mean \pm standard error of mean (SEM). T-test was used to identify any significant difference between two groups. For comparison of multiple groups, 
A

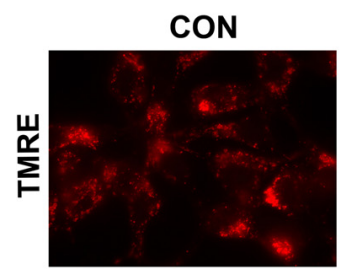

\section{GLUT}

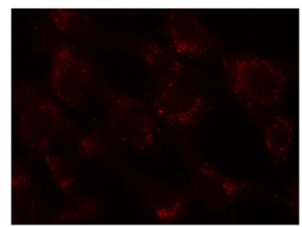

GLUT+ART

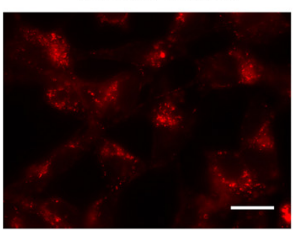

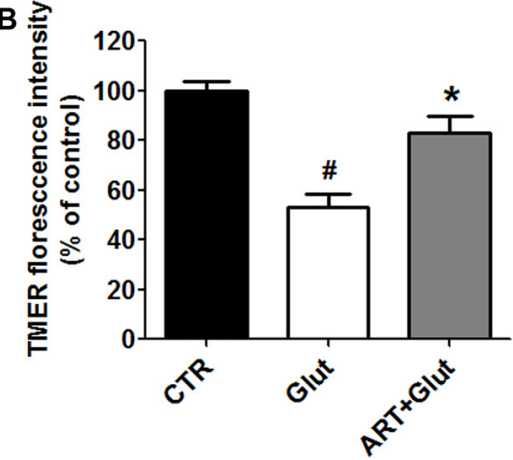

FIGURE 4 | Artemisinin attenuated the glutamate-induced collapse of mitochondrial membrane potential. (A) Tetramethylrhodamine, Ethyl Ester (TMRE) staining showed that artemisinin could reduce glutamate-induced $\Delta \psi \mathrm{m}$ loss. (B) Quantitative data of (A). Scale bar $=20 \mu \mathrm{m}$. ${ }^{\#} p<0.05$ vs. CON group, ${ }^{*} p<0.05$ vs. GLUT group.

A
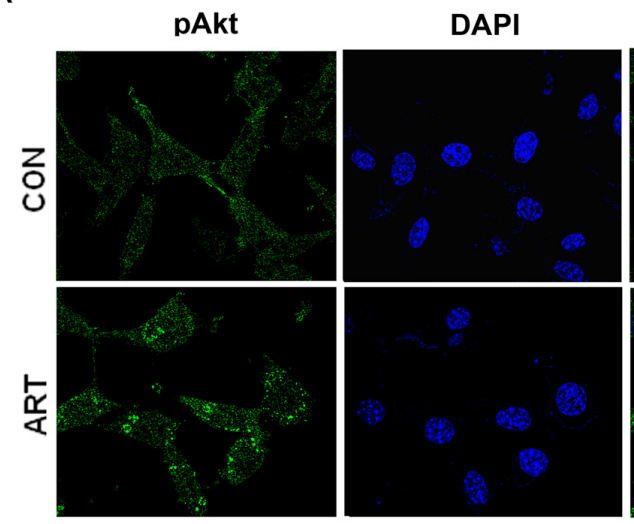

B

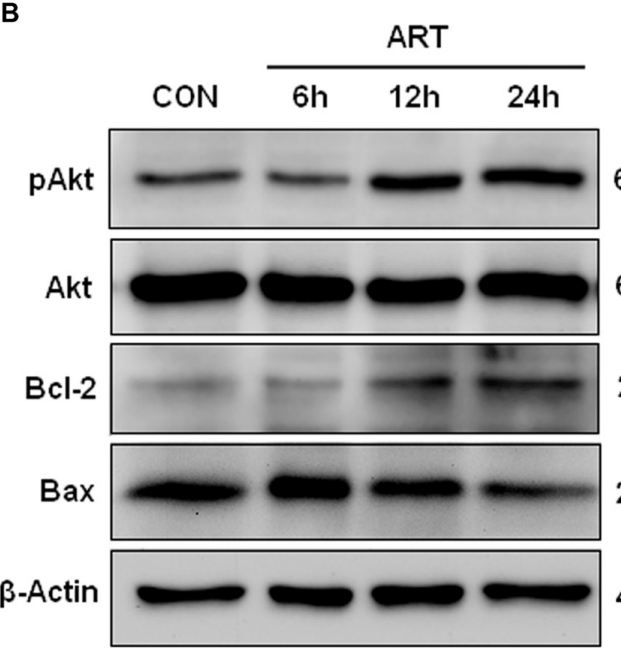

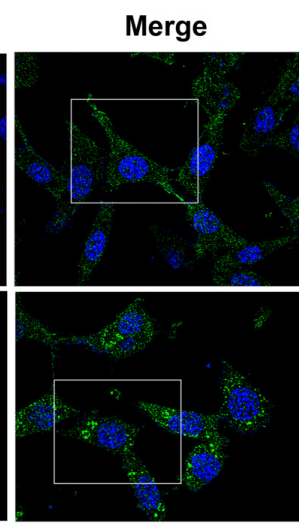
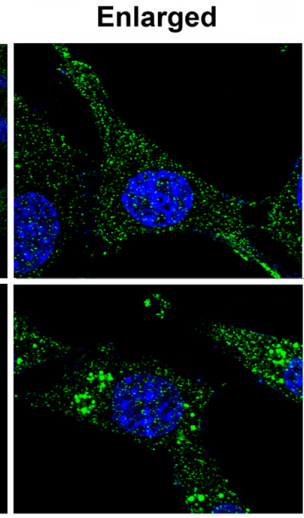

C

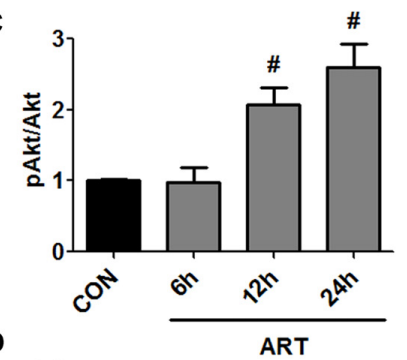

$28 \mathrm{kDa}$

$20 \mathrm{kDa}$

$45 \mathrm{kDa}$

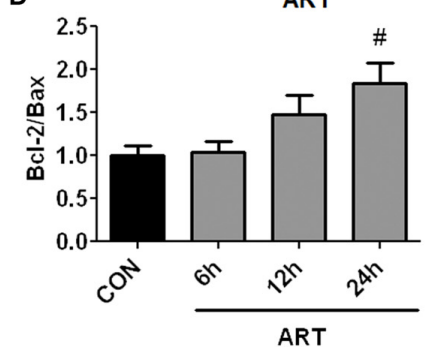

FIGURE 5 | Treatment with artemisinin activated Akt pathway in HT-22 cells. (A) Immunocytochemical staining of phosphor-Akt showed that treatment with artemisinin increased Akt phosphorylation. (B-D) Western blots demonstrated that artemisinin increased pAkt/Akt and Bcl-2/Bax ratio in a time-dependent manner. $\# p<0.05$ vs. CON group.

one-way analysis of variance was used and post hoc Bonferroni For all tests, p-value of less than 0.05 was considered analysis was done to identify the significant differences. significant. 


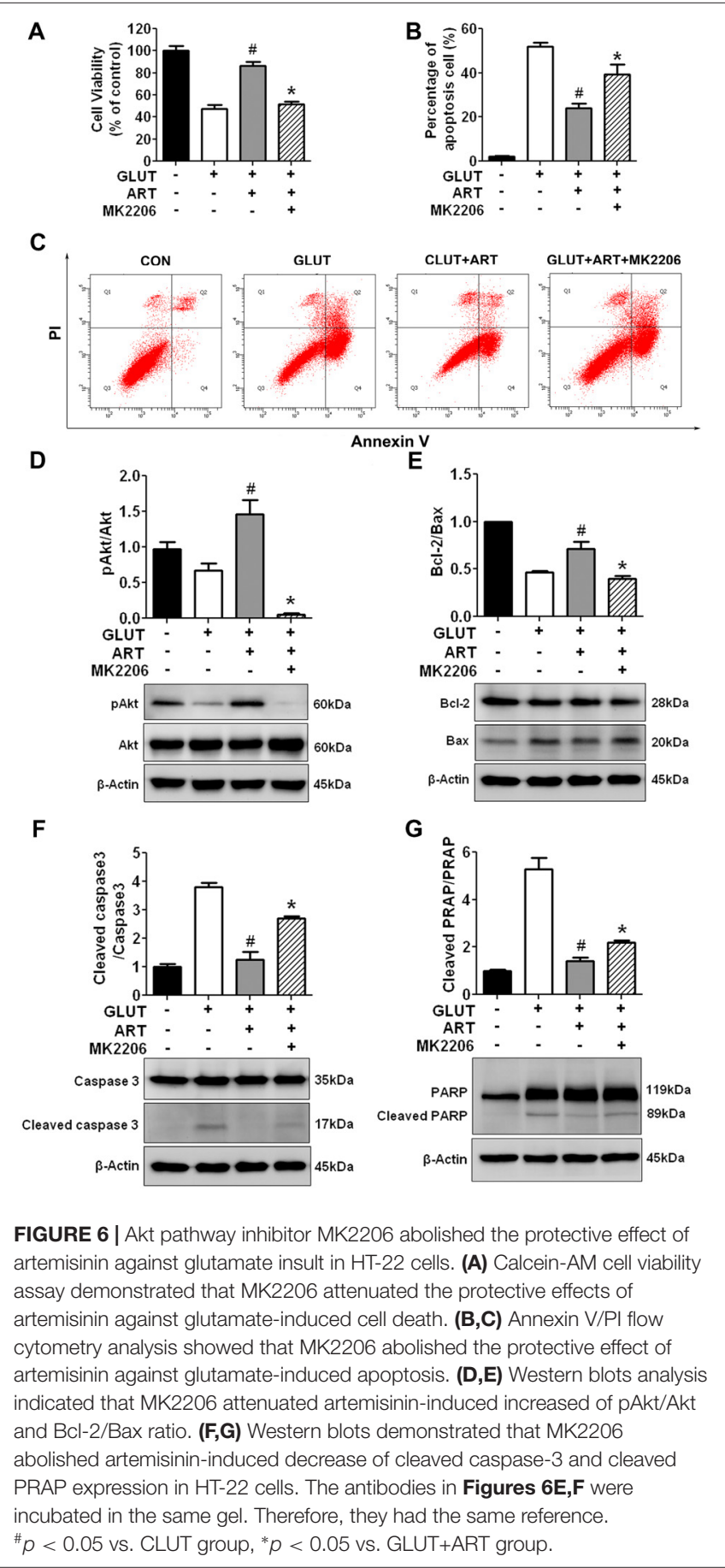

\section{RESULTS}

\section{Artemisinin Pretreatment Reduced Glutamate-Induced Cytotoxicity in HT-22 Cells}

HT-22 cells were treated with various concentrations of artemisinin for $24 \mathrm{~h}$ and cell viability was analyzed by Calcein-AM assay. No cytotoxic effect was observed upon artemisinin treatment in HT-22 cells until reaching $100 \mu \mathrm{M}$ concentration (Figure 1A). The effects of artemisinin on glutamate-induced oxidative stress were evaluated in different treatment paradigms. A dose-dependent neuroprotective effect was indicated when HT-22 cells were pretreated with artemisinin for $12 \mathrm{~h}$ before 12-h glutamate insult (Figures 1B,D). No protective effect against glutamate toxicity was observed when artemisinin was administered less than $12 \mathrm{~h}$ before glutamate insult (Figure 1C). Pretreatment with $25 \mu \mathrm{M}$ artemisinin for $12 \mathrm{~h}$ had an optimum protective effect.

The neuroprotective effect of artemisinin on glutamate insult was further verified by flow cytometry and TUNEL staining. As predicted, glutamate induced a significant increase of apoptosis, which was attenuated by pretreatment of artemisinin (Figure 2).

\section{Protective Effects of Artemisinin on Glutamate-Induced Oxidative Stress and Loss of Mitochondria Membrane Potential}

We determined the effect of artemisinin on glutamate-induced intracellular ROS and mitochondrial ROS production using H2DFFDA and MitoTracker Red CMXRos, respectively. Glutamate significantly increased intracellular ROS and mitochondrial ROS production as compared with control group, which was attenuated upon pretreatment of artemisinin (Figure 3). We further determined the effect of artemisinin pretreatment on mitochondria membrane potential collapse induced by glutamate insult. As predicted, artemisinin reversed glutamate-induced loss of mitochondrial membrane potential evidenced by TMRE analysis (Figure 4).

\section{Akt Signaling Was Involved in the Neuroprotection of Artemisinin}

To determine whether Akt anti-apoptotic pathway was regulated by artemisinin in HT-22 cells, HT-22 cells were incubated with $25 \mu \mathrm{M}$ artemisinin for $24 \mathrm{~h}$ and then processed for the immunocytochemistry of phosphorylated Akt. An increase of phospho-Akt was observed after 24-h treatment of $25 \mu \mathrm{M}$ artemisinin (Figure 5A). Consistently, a time-dependent increase of $\mathrm{pAkt} / \mathrm{Akt}$ and $\mathrm{Bcl}-2 / \mathrm{Bax}$ ratio upon artemisinin treatment was observed by Western Blot (Figures 5B-D).

We further determined whether neuroprotective effect of artemisinin was mediated through Akt signaling. Protective effect of artemisinin on glutamate-induced cell death and apoptosis was negated by co-treatment of Akt inhibitor, $5 \mu \mathrm{M}$ MK2206 (Figures 6A-C). Western blot analysis confirmed that artemisinin-induced increase of pAkt/Akt and Bcl-2/Bax ratio was indeed inhibited with $5 \mu \mathrm{M}$ MK2206 (Figures 6D,E). In addition, MK2206 abolished artemisinin-induced decrease of cleaved caspase-3 and cleaved PARP (Figures 6F,G).

\section{DISCUSSION}

In the current study, we demonstrated that artemisinin prevented neuronal cells from glutamate-induced injury via the activation of Akt pathway. Our study identified a novel neuroprotective 


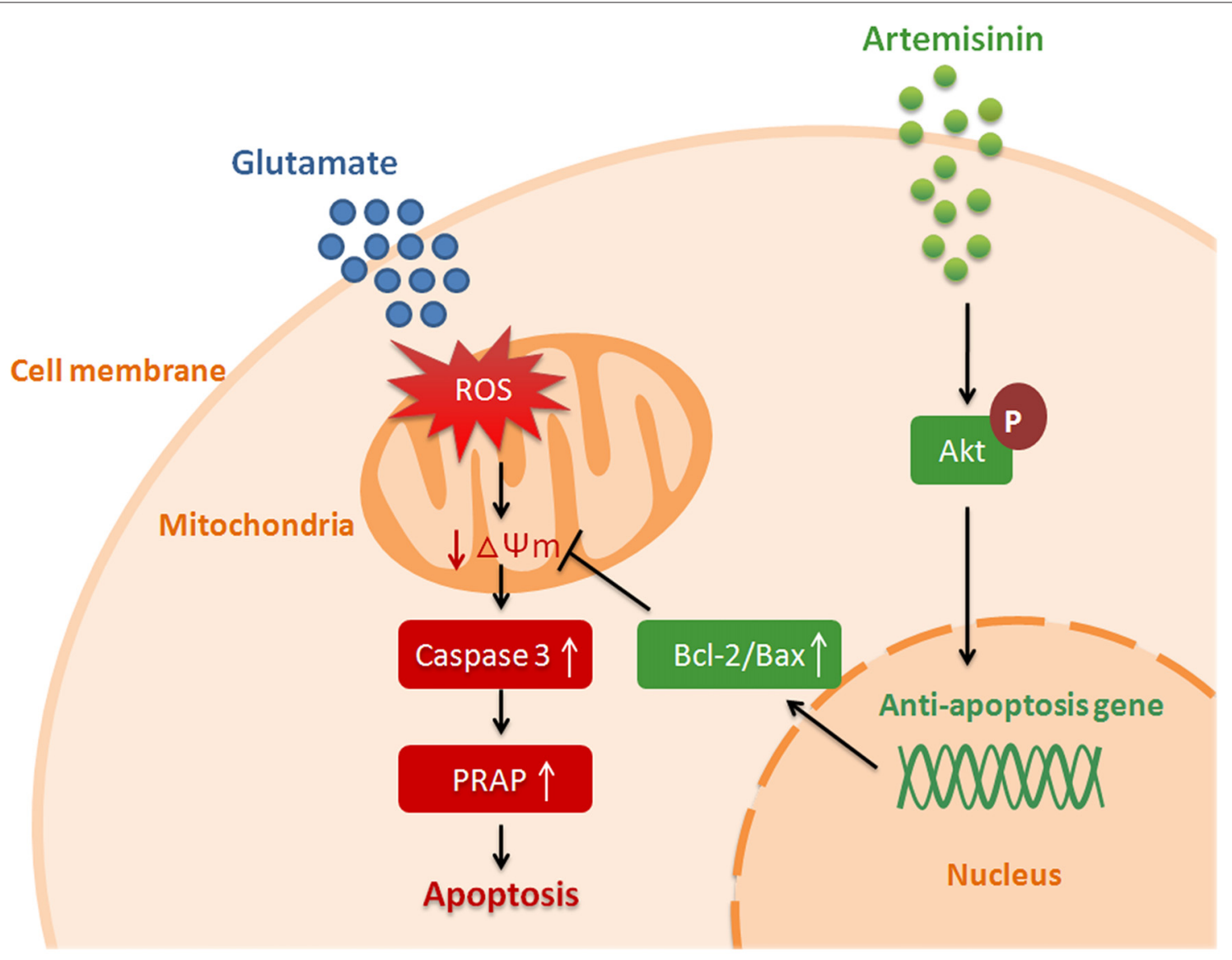

FIGURE 7 | Hypothetical model of artemisinin mediated neuroprotection against glutamate-induced oxidative stress injury.

effect of artemisinin suggesting artemisinin could be potential therapeutic drug for prevention of neurodegenerative disorders.

Anti-malarial effect of artemisinin has been well established. Recently, anti-tumor and anti-inflammation properties have been demonstrated in artemisinin (Zuo et al., 2016). In addition, Zheng et al. (2016) demonstrate that pretreatment with $25 \mu \mathrm{M}$ artemisinin for $1 \mathrm{~h}$ has anti-oxidize effect in nitroprussideinduced oxidative insult in cortical neuron by activating ERK pathway. The hippocampus is one of the most vulnerable parts of brain susceptible to various pathological conditions (Wang et al., 2016). In the current study, we explored the effect of artemisinin on oxidative stress using a HT-22 glutamate model. We found that pretreatment with $25 \mu \mathrm{M}$ artemisinin for $12 \mathrm{~h}$ could provide HT-22 cells protection against glutamateinduced injury. However, pretreatment less than $12 \mathrm{~h}$ failed to protect HT-22 cells from glutamate toxicity. This seems to be a long preprocessing time to pretreat the cell for $12 \mathrm{~h}$. There are several reasons for this phenomenon. First, it has been demonstrated that artemisinin exhibits time-dependent pharmacokinetics (Ashton et al., 1998; Gordi et al., 2002). Short pretreatment period of artemisinin may be insufficient and does not take effect (Zheng et al., 2016). Second, the dose which was used in our study to activate Akt enzymatic activity is much lower than preview study (Steely et al., 2017). Third, dihydroartemisinin is the active metabolite of all artemisinin compounds (Zhao et al., 2012). The metabolic processes of artemisinin to dihydroartemisinin may also contribute long preprocessing time to take effect.

Mitochondria is the main site for ROS production (Wang et al., 2016). We found that pretreatment with artemisinin decreased the subsequent glutamate-induced increase of mitochondrial ROS and total intracellular ROS levels. Accordingly, pretreatment with artemisinin attenuated the glutamate induced mitochondrial membrane potential collapse and rescued HT-22 cells form apoptotic cell death. It has been shown that prior treatment with mild ROS generatros may upregulate expression of hypoxia-inducible factor (HIF) and erythropoietin (Epo), and therefore protect neurons against subsequent ROS stress (Liu et al., 2005). Pretreatment with artemisinin may react with intracellular heme and elicit low levels production of ROS, which could protect cells from lethal ROS insult induced by glutamate damage (Schmuck et al., 2002; Kavishe et al., 2017).

Activation of Akt/Bcl-2 pathway has been demonstrated as an essential anti-apoptotic signaling (Ryou et al., 2013; Cao et al., 2017). The increased Bcl-2/Bax ratio blocks the cytochrome $\mathrm{C}$ 
released from mitochondria, which inhibits the mitochondrial apoptotic pathways (Zhu et al., 2016; Chauhan et al., 2018). The Akt signaling pathway has been indicated as an important drug target of artemisinin (Huang et al., 2013; Ho et al., 2014). The anti-cancer and anti-inflammatory effects of artemisinin have been attributed to the inhibition of Akt signaling pathway (Ho et al., 2014; Luo et al., 2015; Shao et al., 2017). On the other hand, Lee et al. (2012) observed that artemisinin could reduce inflammatory responses in microglial BV2 cells through activation of Akt signaling. In addition, Wang et al. (2015) reported that artemisinin could activate Akt signaling and trigger mitochondrial biogenesis in mice. In the current study, we found that Akt anti-apoptotic pathway was activated by artemisinin in a time dependent manner. Consistently, up-regulation of Bcl-2 and reduction of Bax, cleaved caspase- 3 and cleaved PARP, downstream of Akt activation, were observed upon pretreatment of artemisinin. Furthermore, the protective effect of artemisinin was blocked by MK2206, a highly selective inhibitor of Akt, supporting that activation of Akt pathway was involved in the neuroprotective action of artemisinin.

In summary, our results demonstrated that artemisinin protect neuronal HT-22 cell from glutamate-induced oxidative injury by activation of Akt signaling pathway (Figure 7). Due to its lipid-soluble characteristic, artemisinin can pass BBB and maintain a higher concentration in the central neural system

\section{REFERENCES}

Ashton, M., Hai, T. N., Sy, N. D., Huong, D. X., Van Huong, N., Niêu, N. T., et al. (1998). Artemisinin pharmacokinetics is time-dependent during repeated oral administration in healthy male adults. Drug Metab. Dispos. 26, 25-27.

Cao, Q., Qin, L., Huang, F., Wang, X., Yang, L., Shi, H., et al. (2017). Amentoflavone protects dopaminergic neurons in MPTP-induced Parkinson's disease model mice through PI3K/Akt and ERK signaling pathways. Toxicol. Appl. Pharmacol. 319, 80-90. doi: 10.1016/j.taap.2017.01.019

Chauhan, A. K., Mittra, N., Patel, D. K., and Singh, C. (2018). Cyclooxygenase2 directs microglial activation-mediated inflammation and oxidative stress leading to intrinsic apoptosis in $\mathrm{Zn}$-induced Parkinsonism. Mol. Neurobiol. 55, 2162-2173. doi: 10.1007/s12035-017-0455-0

Cui, L., and Su, X. Z. (2009). Discovery, mechanisms of action and combination therapy of artemisinin. Expert Rev. Anti Infect. Ther. 7, 999-1013. doi: 10.1586/eri.09.68

Gordi, T., Huong, D. X., Hai, T. N., Nieu, N. T., and Ashton, M. (2002). Artemisinin pharmacokinetics and efficacy in uncomplicated-malaria patients treated with two different dosage regimens. Antimicrob. Agents Chemother. 46, 1026-1031. doi: 10.1128/aac.46.4.1026-1031.2002

Guo, Z. (2016). Artemisinin anti-malarial drugs in China. Acta Pharm. Sin. B 6, 115-124. doi: 10.1016/j.apsb.2016.01.008

Haynes, R. K., Chan, W. C., Wong, H. N., Li, K. Y., Wu, W. K., Fan, K. M., et al. (2010). Facile oxidation of leucomethylene blue and dihydroflavins by artemisinins: relationship with flavoenzyme function and antimalarial mechanism of action. ChemMedChem 5, 1282-1299. doi: 10.1002/cmdc. 201000225

Ho, W. E., Peh, H. Y., Chan, T. K., and Wong, W. S. (2014). Artemisinins: pharmacological actions beyond anti-malarial. Pharmacol. Ther. 142, 126-139. doi: 10.1016/j.pharmthera.2013.12.001

Huang, C., Ba, Q., Yue, Q., Li, J., Li, J., Chu, R., et al. (2013). Artemisinin rewires the protein interaction network in cancer cells: network analysis, pathway identification, and target prediction. Mol. Biosyst. 9, 3091-3100. doi: $10.1039 / \mathrm{c} 3 \mathrm{mb} 70342 \mathrm{~h}$

Ismail, H. M., Barton, V., Phanchana, M., Charoensutthivarakul, S., Wong, M. H., Hemingway, J., et al. (2016). Artemisinin activity-based probes identify
(Zuo et al., 2016). As an FDA approval anti-malaria drug, artemisinin has been used in clinic for long-term without apparent adverse effects (Karbwang et al., 1992). Our finding indicates that artemisinin might be a potential novel antioxidant drug for the prevention and treatment of neurodegenerative disorders.

\section{AUTHOR CONTRIBUTIONS}

S-HY designed the experiments. S-PL, WL, AW and RL conducted the experiments. S-PL and S-HY wrote the manuscript. All the authors analyzed the data, revised the manuscript and approved the final manuscript.

\section{FUNDING}

This work was partly supported by the National Natural Science Foundation of China (Grant No. 81641088), the Natural Science Foundation of Guangdong Province (Grant No. 2016A030310267), the Science and Technology Planning Project of Guangdong Province (Grant No. 2015A030302091), the Science and Technology Planning Project of Guangzhou (Grant No. 201607010160), the Nature Science Foundation in Guangzhou Medical University (Grant No. 2015C14) and the Key Medical Disciplines and Specialities Program of Guangzhou.

multiple molecular targets within the asexual stage of the malaria parasites Plasmodium falciparum 3D7. Proc. Natl. Acad. Sci. U S A 113, 2080-2085. doi: $10.1073 /$ pnas. 1600459113

Karbwang, J., Sukontason, K., Rimchala, W., Namsiripongpun, W., Tin, T., Auprayoon, P., et al. (1992). Preliminary report: a comparative clinical trial of artemether and quinine in severe falciparum malaria. Southeast Asian J. Trop. Med. Public Health 23, 768-772.

Kavishe, R. A., Koenderink, J. B., and Alifrangis, M. (2017). Oxidative stress in malaria and artemisinin combination therapy: Pros and Cons. FEBS J. 284, 2579-2591. doi: 10.1111/febs.14097

Kim, W. S., Choi, W. J., Lee, S., Kim, W. J., Lee, D. C., Sohn, U. D., et al. (2015). anti-inflammatory, antioxidant and antimicrobial effects of artemisinin extracts from artemisia annua L. Korean J. Physiol. Pharmacol. 19, 21-27. doi: 10.4196/kjpp.2015.19.1.21

Lee, I. S., Ryu, D. K., Lim, J., Cho, S., Kang, B. Y., and Choi, H. J. (2012). Artesunate activates Nrf2 pathway-driven anti-inflammatory potential through ERK signaling in microglial BV2 cells. Neurosci. Lett. 509, 17-21. doi: 10.1016/j. neulet.2011.12.034

Lin, S. P., Ye, S., Long, Y., Fan, Y., Mao, H. F., Chen, M. T., et al. (2016). Circular RNA expression alterations are involved in OGD/R-induced neuron injury. Biochem. Biophys. Res. Commun. 471, 52-56. doi: 10.1016/j.bbrc.2016. 01.183

Liu, J., Narasimhan, P., Yu, F., and Chan, P. H. (2005). Neuroprotection by hypoxic preconditioning involves oxidative stress-mediated expression of hypoxiainducible factor and erythropoietin. Stroke 36, 1264-1269. doi: 10.1161/01.str. 0000166180.91042 .02

Luo, Q., Lin, J., Zhang, L., Li, H., and Pan, L. (2015). The anti-malaria drug artesunate inhibits cigarette smoke and ovalbumin concurrent exposureinduced airway inflammation and might reverse glucocorticoid insensitivity. Int. Immunopharmacol. 29, 235-245. doi: 10.1016/j.intimp.2015.11.016

Ryou, M. G., Choudhury, G. R., Li, W., Winters, A., Yuan, F., Liu, R., et al. (2015). Methylene blue-induced neuronal protective mechanism against hypoxiareoxygenation stress. Neuroscience 301, 193-203. doi: 10.1016/j.neuroscience. 2015.05.064

Ryou, M. G., Choudhury, G. R., Winters, A., Xie, L., Mallet, R. T., and Yang, S. H. (2013). Pyruvate minimizes rtPA toxicity from in vitro oxygen-glucose 
deprivation and reoxygenation. Brain Res. 1530, 66-75. doi: 10.1016/j.brainres. 2013.07.029

Schmuck, G., Roehrdanz, E., Haynes, R. K., and Kahl, R. (2002). Neurotoxic mode of action of artemisinin. Antimicrob. Agents Chemother. 46, 821-827. doi: 10.1128/aac.46.3.821-827.2002

Shao, Y. Y., Zhang, T. L., Wu, L. X., Zou, H. C., Li, S., Huang, J., et al. (2017). AKT Axis, miR-21 and RECK play pivotal roles in dihydroartemisinin killing malignant glioma cells. Int. J. Mol. Sci. 18:E350. doi: 10.3390/ijms 18020350

Sozen, E., and Ozer, N. K. (2017). Impact of high cholesterol and endoplasmic reticulum stress on metabolic diseases: an updated mini-review. Redox Biol. 12, 456-461. doi: 10.1016/j.redox.2017.02.025

Steely, A. M., Willoughby, J. A. Sr., Sundar, S. N., Aivaliotis, V. I., and Firestone, G. L. (2017). Artemisinin disrupts androgen responsiveness of human prostate cancer cells by stimulating the $26 \mathrm{~S}$ proteasome-mediated degradation of the androgen receptor protein. Anticancer Drugs 28, 1018-1031. doi: $10.1097 / \mathrm{cad} .0000000000000547$

Vida, C., de Toda, I. M., Cruces, J., Garrido, A., Gonzalez-Sanchez, M., and De La Fuente, M. (2017). Role of macrophages in age-related oxidative stress and lipofuscin accumulation in mice. Redox Biol. 12, 423-437. doi: 10.1016/j.redox. 2017.03.005

Wang, D. T., He, J., Wu, M., Li, S. M., Gao, Q., and Zeng, Q. P. (2015). Artemisinin mimics calorie restriction to trigger mitochondrial biogenesis and compromise telomere shortening in mice. PeerJ 3:e822. doi: 10.7717/peerj.822

Wang, Z., Ye, Z., Huang, G., Wang, N., Wang, E., and Guo, Q. (2016). Sevoflurane post-conditioning enhanced hippocampal neuron resistance to global cerebral ischemia induced by cardiac arrest in rats through PI3K/Akt survival pathway. Front. Cell. Neurosci. 10:271. doi: 10.3389/fncel.2016.00271

Xie, L., Li, W., Winters, A., Yuan, F., Jin, K., and Yang, S. (2013). Methylene blue induces macroautophagy through $5^{\prime}$ adenosine monophosphate-activated protein kinase pathway to protect neurons from serum deprivation. Front. Cell. Neurosci. 7:56. doi: 10.3389/fncel.2013.00056
Yan, F., Wang, H., Gao, Y., Xu, J., and Zheng, W. (2017). Artemisinin protects retinal neuronal cells against oxidative stress and restores rat retinal physiological function from light exposed damage. ACS Chem. Neurosci. 8, 1713-1723. doi: 10.1021/acschemneuro.7b00021

Zhao, Y. G., Wang, Y., Guo, Z., Gu, A. D., Dan, H. C., Baldwin, A. S., et al. (2012). Dihydroartemisinin ameliorates inflammatory disease by its reciprocal effects on Th and regulatory $\mathrm{T}$ cell function via modulating the mammalian target of rapamycin pathway. J. Immunol. 189, 4417-4425. doi: 10.4049/jimmunol. 1200919

Zheng, W., Chong, C. M., Wang, H., Zhou, X., Zhang, L., Wang, R., et al. (2016). Artemisinin conferred ERK mediated neuroprotection to PC12 cells and cortical neurons exposed to sodium nitroprusside-induced oxidative insult. Free Radic. Biol. Med. 97, 158-167. doi: 10.1016/j.freeradbiomed.2016.05.023

Zhu, H., Zhang, Y., Shi, Z., Lu, D., Li, T., Ding, Y., et al. (2016). The neuroprotection of liraglutide against ischaemia-induced apoptosis through the activation of the PI3K/AKT and MAPK pathways. Sci. Rep. 6:26859. doi: $10.1038 /$ srep26859

Zuo, S., Li, Q., Liu, X., Feng, H., and Chen, Y. (2016). The potential therapeutic effects of artesunate on stroke and other central nervous system diseases. Biomed Res. Int. 2016:1489050. doi: 10.1155/2016/1489050

Conflict of Interest Statement: The authors declare that the research was conducted in the absence of any commercial or financial relationships that could be construed as a potential conflict of interest.

Copyright (c) 2018 Lin, Li, Winters, Liu and Yang. This is an open-access article distributed under the terms of the Creative Commons Attribution License (CC BY). The use, distribution or reproduction in other forums is permitted, provided the original author(s) and the copyright owner are credited and that the original publication in this journal is cited, in accordance with accepted academic practice. No use, distribution or reproduction is permitted which does not comply with these terms. 